DFTT 54/95

Torino, 1995

\title{
Color dipole systematics of diffractive photo- and electroproduction of vector mesons
}

\author{
J.Nemchik ${ }^{a, b}$, N.N. Nikolaev ${ }^{c, d}$, E. Predazzi ${ }^{a}$, B.G. Zakharov ${ }^{a, d}$ \\ ${ }^{a}$ Dipartimento di Fisica Teorica, Università di Torino, \\ and INFN, Sezione di Torino, I-10125, Torino, Italy \\ ${ }^{b}$ Institute of Experimental Physics, Slovak Academy of Sciences, \\ Watsonova 47, 04353 Kosice, Slovak Republik \\ ${ }^{c} I K P($ Theorie), KFA Jülich, 5170 Jülich, Germany \\ ${ }^{d}$ L. D. Landau Institute for Theoretical Physics, GSP-1, 117940, \\ ul. Kosygina 2, Moscow 117334, Russia.
}

\begin{abstract}
We present the first evaluation of color dipole cross section from experimental data on diffractive photo- and electroproduction of vector mesons. The dipole-size and energy dependence of the found dipole cross section is consistent with expectations from the BFKL dynamics.
\end{abstract}

E-mail: nemchik@to.infn.it 
In the color dipole picture of high energy scattering [1, 2, 3, 4, the dipole cross section is as a fundamental quantity as the low $x$ gluon structure function of the proton in the conventional parton model. The diffractive electroproduction of vector mesons

$$
\gamma^{*} p \rightarrow V p, \quad V=\rho^{0}, \omega^{0}, \phi^{0}, J / \Psi, \Upsilon
$$

offers a unique window on the dipole cross section [5, 6, 7]. Here the crucial point is that for the shrinkage of the transverse size of the virtual photon with the virtuality $Q^{2}$, the $1 S$ vector meson production amplitude probes the dipole cross section at the dipole size $r \sim r_{S}$, where the scanning radius equals [8, 9]

$$
r_{S}=\frac{A}{\sqrt{m_{V}^{2}+Q^{2}}}
$$

with $A=6$. Specifically, the amplitudes of production of the transversely $(\mathrm{T})$ and longitudinally (L) polarized $1 S$ vector mesons are of the form [8, 9] $\mathcal{M}_{T} \propto r_{S}^{2} \sigma\left(x_{e f f}, r_{S}\right)$, $\mathcal{M}_{L} \approx \frac{\sqrt{Q^{2}}}{m_{V}} \mathcal{M}_{T}$, and one can extract the dipole cross section from the vector meson production cross sections. For the alternative description of vector meson production at very large $Q^{2}$ in terms of the gluon structure function of the proton see 110, 11].

In the present communication we report the results of the first evaluation of the color dipole cross section from the experimental data on real photoproduction and electroproduction of the $\rho^{0}, \phi^{0}$ and $J / \Psi$ from the fixed target and HERA collider experiments. A nice consistency with color blindness of the dipole cross section is found. When one takes the same value of the scanning radius, the $\rho^{0}, \phi^{0}$ and $J / \Psi$ production data yield close values of the dipole cross section. The results of the HERA experiments allow the first evaluation of energy dependence of the dipole cross section at different dipole sizes and confirm the prediction from BFKL dynamics that the smaller is the dipole size the faster is the rise of the dipole cross section [3, 12].

One needs the probability amplitudes to find color dipole of size $r$ in the photon and vector meson. Here we use the formalism introduced in [1, 5] and expounded in [9]. The spin independence of the dipole cross section leads to the $s$-channel helicity conservation. In terms of the lightcone "radial" wave function $\phi(r, z)$ of the $q \bar{q}$ Fock state of the vector meson and the color dipole cross section $\sigma(\nu, r)$, the imaginary part of the forward production 
amplitudes equals

$$
\begin{gathered}
\operatorname{Im} \mathcal{M}_{T}\left(x_{e f f}, Q^{2}\right)=\frac{N_{c} C_{V} \sqrt{4 \pi \alpha_{e m}}}{(2 \pi)^{2}} \cdot \\
\cdot \int d^{2} \mathbf{r} \sigma\left(x_{e f f}, r\right) \int_{0}^{1} \frac{d z}{z(1-z)}\left\{m_{q}^{2} K_{0}(\varepsilon r) \phi(r, z)-\left[z^{2}+(1-z)^{2}\right] \varepsilon K_{1}(\varepsilon r) \partial_{r} \phi(r, z)\right\} \\
=\frac{1}{\left(m_{V}^{2}+Q^{2}\right)^{2}} \int \frac{d r^{2}}{r^{2}} \frac{\sigma\left(x_{e f f}, r\right)}{r^{2}} W_{T}\left(Q^{2}, r^{2}\right)=g_{T} \sqrt{4 \pi \alpha_{e m}} C_{V} \sigma\left(x_{e f f}, r_{S}\right) \frac{m_{V}^{2}}{m_{V}^{2}+Q^{2}} \cdot \\
\cdot \int d^{2} \mathbf{r} \sigma\left(x_{e f f}, r\right) \int_{0}^{1} d z\left\{\left[m_{q}^{2}+z(1-z) m_{V}^{2}\right] K_{0}(\varepsilon r) \phi(r, z)-\varepsilon K_{1}(\varepsilon r) \partial_{r} \phi(r, z)\right\} \\
=\frac{\operatorname{Im}_{L}\left(x_{e f f}, Q^{2}\right)=\frac{N_{c} C_{V} \sqrt{4 \pi \alpha_{e m}}}{(2 \pi)^{2}} \frac{\sqrt{Q^{2}}}{m_{V}} \cdot}{m_{V}\left(m_{V}^{2}+Q^{2}\right)^{2}} \int \frac{d r^{2}}{r^{2}} \frac{\sigma\left(x_{e f f}, r\right)}{r^{2}} W_{L}\left(Q^{2}, r^{2}\right)=g_{L} \sqrt{4 \pi \alpha_{e m}} C_{V} \sigma\left(x_{e f f}, r_{S}\right) \frac{\sqrt{Q^{2}}}{m_{V}} \cdot \frac{m_{V}^{2}}{m_{V}^{2}+Q^{2}},
\end{gathered}
$$

where $\mathbf{r}$ is the transverse $q \bar{q}$ separation in the lightcone meson, i.e., the dipole size, $z$ is the fraction of the momentum of the meson carried by the quark,

$$
\varepsilon^{2}=m_{q}^{2}+z(1-z) Q^{2}
$$

$N_{c}=3$ is the number of colors, $C_{V}=\frac{1}{\sqrt{2}}, \frac{1}{3 \sqrt{2}}, \frac{1}{3}, \frac{2}{3}$ for the $\rho^{0}, \omega^{0}, \phi^{0}, J / \Psi$ production, respectively, $K_{0,1}(x)$ is the modified Bessel function. In (3),(4) the dipole cross section enters at an effective value of the Bjorken variable

$$
x_{e f f}=\frac{Q^{2}+m_{V}^{2}}{2 m_{p} \nu} .
$$

The normalization of production amplitudes is such that

$$
\left.\frac{d \sigma}{d t}\right|_{t=0}=\frac{|\mathcal{M}|^{2}}{16 \pi}
$$

Eqs. (3),(4) give the imaginary part of production amplitudes, one can easily include small corrections for the real part by the substitution [13]

$$
\sigma\left(x_{e f f}, r\right) \Longrightarrow\left(1-i \cdot \frac{\pi}{2} \cdot \frac{\partial}{\partial \log x_{e f f}}\right) \sigma\left(x_{e f f}, r\right)=\left[1-i \cdot \alpha_{V}\left(x_{e f f}, r\right)\right] \sigma\left(x_{e f f}, r\right)
$$

Many gluon containing higher Fock states $q \bar{q} g \ldots$ are very important at high energy $\nu$. The crucial point is that in leading $\log \frac{1}{x}$ their effect can be reabsorbed into the energy dependence of $\sigma(x, r)$, which satisfies the generalized BFKL equation ( [2, 3], for the related approach see also [4]). The color dipole factorization formulas (3),(4) follow from diagonalization of the 
scattering matrix in the $(\mathbf{r}, z)$ representation. Clearly, this factorization holds even when the dipole size is large, beyond the perturbative region of small sizes. Only the wave function of the lowest $q \bar{q}$ Fock state enters color dipole factorization (3),(4) formulas. At large dipole size $r$ one can identify $\phi(r, z)$, which is the probability amplitude to find the dipole of size $r$, with the constituent quark wave function of the meson and to develop a viable phenomenology of diffractive scattering which is purely perturbative for small size mesons and allows a sensible interpolation between soft interactions for large dipoles and hard perturbative interactions of small dipoles. Here the key property is the flavour independence of the pomeron exchange dipole cross section. The detailed description of parameterization of $\phi(r, z)$, which incorporates the hard-QCD short distance behaviour, is given in [9]. The parameters of the wave function were constrained to reproduce the generally accepted radii of vector mesons and the width of leptonic decays $V \rightarrow e^{+} e^{-}$, they are listed in Table 1 .

Eqs. (3), (4) describe the pure pomeron exchange, which dominates at large values of the Regge parameter $\omega=1 / x_{\text {eff }}$. At moderate values of $\omega=1 / x_{\text {eff }}$ a substantial part of the $\gamma p$ total cross section is due to the non-vacuum Reggeon exchange contribution. For instance, the Regge fit to the $\gamma p$ total cross section can be cast in the form [14]

$$
\sigma_{t o t}(\gamma p)=\sigma_{\mathbf{I P}}(\gamma p) \cdot\left(1+\frac{A}{\omega^{\Delta}}\right)
$$

where the term $A / \omega^{\Delta}$ in the factor $f=1+A / \omega^{\Delta}$ represents the non-vacuum Reggeon exchange contribution, the Donnachie-Landshoff fit gives $A=2.332$ and $\Delta=0.533$. The similar Regge correction emerges also in real $\rho^{0}$ photoproduction amplitude. We do not know how large this non-vacuum contribution to the $\rho^{0}$ production is at large $Q^{2}$, for the crude estimation we assume that the Reggeon/pomeron ratio scales with $\omega$, which is not inconsistent with the known decomposition of the proton structure function into the valence (non-vacuum Reggeon) and sea (pomeron) contributions. Then, for the NMC kinematics we find $f=1.25$ at $\omega \simeq 70$ relevant to $Q^{2}=3 \mathrm{GeV}^{2}$ and $f=1.8$ at $\omega \simeq 9$ relevant to $Q^{2}=20 \mathrm{GeV}^{2}$. For the HERA energy range the non-vacuum Reggeon exchange contribution can be neglected due to a large value of the Regge parameter $\omega$. In practical terms, we calculate the quantity $d \sigma\left(\gamma^{*} \rightarrow V\right) /\left.d t\right|_{t=0}=f^{2} \cdot d \sigma_{\mathbf{I P}}\left(\gamma^{*} \rightarrow V\right) /\left.d t\right|_{t=0}$. For the Zweig rule, one expects $f=1$ for the $\phi^{0}, J / \Psi, \Upsilon$ production.

In the final form of amplitudes in (3),(4) we separated out the rapid dependence on $Q^{2}$ 
and/or scanning radius $r_{S}$, and the so introduced coefficient functions $g_{T, L}$ are smooth functions of $Q^{2}$. The possibility of such a local relationship between the production amplitude and the dipole cross section at a well defined dipole size $r_{S}$ is based on two observations: i) the weight functions $W_{T, L}\left(Q^{2}, r^{2}\right)$ have a sharp peak at $r=A_{T, L} / \sqrt{Q^{2}+m_{V}^{2}}$ with $A_{T, L} \sim 6$ [9], ii) the ratio $\sigma\left(x_{e f f}, r\right) / r^{2}$ is a smooth function of the radius [3, 12]. For these reasons, the coefficient functions $g_{T, L}$ have only a weak sensitivity to the detailed shape of the dipole cross section. The gross features of $\sigma\left(x_{e f f}, r\right)$ are well understood and in [15] good quantitative description of the small- $x$ structure function of the proton was obtained in the color dipole BFKL dynamics. The model dependence of $g_{T, L}$ can be evaluated using the low-energy and high-energy forms of $\sigma\left(x_{e f f}, r\right)$ described in [15, 9], which have a markedly different $r$ dependence. In Fig. 1 we present the $Q^{2}$ dependence of $g_{T, L}$ for different production processes at $W=15 \mathrm{GeV}$ and $W=150 \mathrm{GeV}$ (values of interest for the fixed target and HERA experiments respectively). The variation of the resulting coefficient functions $g_{T, L}$ from small to large $W$ does not exceed $15 \%$, which is a conservative estimate of the theoretical uncertainty of the above procedure. The residual smooth $Q^{2}$ dependence of $g_{T, L}$ mostly reflects the smooth and well understood $Q^{2}$ dependence of the scale factors $A_{T, L}$ which enter the relationship between the position $A_{T, L} / \sqrt{Q^{2}+m_{V}^{2}}$ of the peak of $W_{T, L}\left(Q^{2}, r^{2}\right)$ and $r_{S}$ as given by Eq. (2) (see also the discussion in [9]). In (3),(4), the $g_{T, L}$ are so defined as to relate the amplitude to $\sigma\left(x_{e f f}, r_{S}\right)$ at the well defined scanning radius (2), reabsorbing the effect of small departure of $A_{T, L}$ from 6 into $g_{T, L}$.

The experimentally measured forward cross production section section equals

$$
\left.\frac{d \sigma\left(\gamma^{*} \rightarrow V\right)}{d t}\right|_{t=0}=\frac{f^{2}}{16 \pi} \cdot\left[\left(1+\alpha_{V, T}^{2}\right) \mathcal{M}_{T}^{2}+\epsilon\left(1+\alpha_{V, L}^{2}\right) \mathcal{M}_{L}^{2}\right]
$$

The difference between $\alpha_{V, L}$ and $\alpha_{V, T}$ for the longitudinal and transverse cross sections and the overall effect of the real part is marginal and can safely be neglected compared to other uncertainties. Then, making use of the so determined $g_{T, L}$, in terms of the experimentally measured forward production cross section we can write using Eq. (3), (4), (7) and (10)

$$
\begin{aligned}
& \sigma\left(x_{e f f}, r_{S}\right)=\frac{1}{f} \cdot \frac{1}{C_{V}} \cdot \frac{Q^{2}+m_{V}^{2}}{m_{V}^{2}} \cdot \frac{2}{\sqrt{\alpha_{e m}}} \cdot\left(g_{T}^{2}+\epsilon g_{L}^{2} \cdot \frac{Q^{2}}{M_{V}^{2}}\right)^{-1 / 2} \\
& \cdot\left(1+\alpha_{V}^{2}\right)^{-1 / 2} \sqrt{\left.\frac{d \sigma\left(\gamma^{*} \rightarrow V\right)}{d t}\right|_{t=0}}
\end{aligned}
$$


Here $\epsilon$ is the longitudinal polarization of the photon the values of which are taken from the corresponding experimental publications. In (11) $f$ is the above discussed factor which accounts for the non-vacuum Reggeon contribution to the $\rho^{0}$ production, for the $\phi^{0}$ and $J / \Psi$ production, $f \equiv 1$. In the case the experimental data are presented in the form of the $t$-integrated cross section, we evaluate $\left.\frac{d \sigma\left(\gamma^{*} \rightarrow V\right)}{d t}\right|_{t=0}=B \sigma_{t o t}\left(\gamma^{*} \rightarrow V\right)$ using the diffraction slope $B$ as cited in the same publication. In Fig. 2 we show the results of such analysis of the low energy [16] and ZEUS [17] data on real photoproduction of the $\phi^{0}$, the NMC data [18] on electroproduction of the $\rho^{0}$ and $\phi^{0}$, the HERA results on real and virtual photoproduction of the $\rho^{0}$ (H1 [19], ZEUS [20, 21, 22]), fixed target data on real photoproduction [23, 24] and electroproduction [25] of the $J / \Psi$ and HERA results on real photoproduction of the $J / \Psi$ (H1 [26], ZEUS [27]). The error bars shown here correspond to the error bars in the measured cross sections as cited in the experimental publications. The experimental data on vector meson production fall into the two broad categories: the fixed target data taken at typical center of mass energy $W \sim(10-15) \mathrm{GeV}$ and the HERA collider data taken at $W \sim(70-150) \mathrm{GeV}$. The color dipole cross section is flavour blind, there is only kinematical dependence on the vector meson through the definition of $x_{e f f}$. However, if different reactions are compared at the same value of the scanning radius $r_{S}$, i.e., at the same value of $Q^{2}+m_{V}^{2}$, then at the fixed energy $\nu$ the corresponding values of $x_{e f f}$ are equal. Consequently, we expect that within each data group, the procedure (11) applied to different vector mesons will yield the same value of $\sigma\left(x_{e f f}, r_{S}\right)$ at the same value of $r_{S}$. This is an important consistency check. The experimental data on the vector meson production give a solid evidence for the decrease of the dipole cross section towards small dipole size $r_{S}$. The fixed target data exhibit a decrease of $\sigma\left(x_{e f f}, r_{S}\right)$ by one order in the magnitude from $r_{S} \approx 1.2 \mathrm{fm}$ in real photoproduction of the $\phi^{0}$ down to $r_{S} \approx 0.24 \mathrm{fm}$ in electroproduction of the $\rho^{0}$ at $Q^{2}=23 \mathrm{GeV}^{2}$ and of the $J / \Psi$ at $Q^{2}=13 \mathrm{GeV}^{2}$. In the region of overlapping values of $r_{S}$ there is a remarkable consistency between the dipole size dependence and also absolute values of the dipole cross section determined from the data on the $\rho^{0}, \phi^{0}$ and $J / \Psi$ production, in agreement with the flavour independence of the dipole cross section.

The high energy data from HERA exhibit a decrease of the dipole cross section by the factor $\sim 6-7$ from $r_{S}=1.5 \mathrm{fm}$ in real photoproduction of the $\rho^{0}$ down to $r_{S}=0.26 \mathrm{fm}$ in electroproduction of the $\rho^{0}$ at $Q^{2}=19.5 \mathrm{GeV}^{2}$. A comparison of the fixed-target and 
HERA data on real photoproduction and electroproduction confirms the prediction [3, 12, 9 of faster growth of the dipole cross section at smaller dipole size, although the error bars are still large.

The above determination of $\sigma\left(x_{e f f}, r_{S}\right)$ is rather crude for the several reasons. i) First, the early EMC data on vector meson production are well known to have been plagued by a background from the inelastic process $\gamma^{*} p \rightarrow V X$. A comparison of the more recent NMC [18] and the EMC data [28] on the $\rho^{0}$ production suggests that the inelastic admixture could have enhanced the observed cross section by as large factor as $\sim 3$ at $Q^{2}=17 \mathrm{GeV}^{2}$. Such an uncertainty would have resulted in overestimation of $\sigma\left(x_{e f f}, r_{S}\right)$ by the factor $\sim 1.8$. This may be an origin of slightly larger values of $\sigma\left(x_{e f f}, r_{S}\right)$ deduced from the EMC data 25 on the $J / \Psi$ electroproduction. Also the scattering of the measured $J / \Psi$ photoproduction cross sections is quite large, $\sim 50 \%$. Still, this factor of 2 uncertainty is much smaller than the more than one order in magnitude variation of $\sigma\left(x_{e f f}, r_{S}\right)$ over the considered span in $r_{S}$. In the recent NMC data [18] a special care has been taken to eliminate an inelastic background and the values of $\sigma\left(x_{e f f}, r_{S}\right)$ from the $\rho^{0}$ and $\phi^{0}$ production data are consistent within the experimental error bars.

ii) There are further uncertainties with the value of the diffraction slope $B$. At large $Q^{2}$, the values of $B$ could have been underestimated due to the same inelastic background. Even in the better quality data, there are uncertainties with extrapolation down to $t=0$. Because of the curvature of the diffraction cone, one may somewhat underestimate the forward cross section. The experimental situation with the diffraction slopes is quite unsatisfactory, in the case of the $J / \Psi$ and at large $Q^{2}$ for light vector mesons even the $\sim 50 \%$ uncertainty can not be excluded at the moment. However, this uncertainty in the diffraction slope corresponds to $\lesssim 25 \%$ uncertainty in our evaluation of $\sigma\left(x_{e f f}, r_{S}\right)$, which is sufficient for the purposes of the present exploratory evaluation of $\sigma\left(x_{e f f}, r_{S}\right)$.

iii) There is also the above evaluated conservative $\lesssim 15 \%$ theoretical inaccuracy of our procedure.

iv) Finally, there is a residual uncertainty with the wave function of light vector mesons. As a matter of fact, if the dipole cross section were known, then diffractive $\gamma^{*} p \rightarrow V p$ must be regarded as a local probe of the wave function of vector mesons at $r \approx r_{S}$ [7], which may eventually become one of applications of vector meson production. To this end, the 
consistency of $\sigma\left(x_{e f f}, r\right)$ determined from different reactions indicates that wave functions of vector mesons are reasonably constrained by the leptonic width. Here we only wish to notice, that at high $Q^{2}$ and small scanning radius, the vector meson production cross section is $\propto \Gamma\left(V \rightarrow e^{+} e^{-}\right)$and even the factor 2 uncertainty in this quantity corresponds to only $\lesssim 40 \%$ uncertainty in the determination of the dipole cross section.

To summarize, within the above stated uncertainties of our simple procedure and the experimental error bars, there is a consistency between the dipole cross section determined from the $\rho^{0} \phi^{0}$ and $J / \Psi$ production data. This is the first direct determination of the dipole cross section from the experimental data and our main conclusions on properties of the dipole cross section are not affected by the above cited uncertainties.

In Fig. 2 we show also the dipole cross section from the BFKL analysis 15, 9], which gives a good quantitative description of structure function of the photon at small $x$. We conclude that the color dipole BFKL dynamics provides a unified description of the experimental data on diffractive production of vector mesons and on the proton structure function.

Conclusions. We presented the first determination of the dipole cross section from the experimental data on diffractive production of vector mesons. We evaluated the color dipole cross section $\sigma\left(x_{e f f}, r_{S}\right)$ at dipole size $r_{S}$ down to $r_{S} \approx 0.2 \mathrm{fm}$ and confirmed the theoretically expected rapid decrease of $\sigma\left(x_{e f f}, r_{S}\right)$ towards small $r_{S}$. We found a remarkable consistency between the absolute value and the dipole size and energy dependence of the dipole cross section extracted from the data on different vector mesons. This constitutes an important cross-check of the color dipole picture of the QCD pomeron. The found pattern of the energy dependence of the dipole cross section is consistent with the flavour independence and with expectations from the BFKL dynamics.

\section{Acknowledgements:}

This work was partly supported by the INTAS grant No. 93-239. 


\section{References}

[1] N.N. Nikolaev and B.G. Zakharov, Z. Phys. C49 (1991) 607; Z. Phys. C53 (1992) 331;

[2] N.Nikolaev and B.G.Zakharov, JETP 78 (1994) 598; Z. Phys. C64 (1994)631;

[3] N.N.Nikolaev, B.G.Zakharov and V.R.Zoller, JETP Letters 59 (1994) 8; JETP 78 (1994) 866; Phys. Lett. B328 (1994) 486.

[4] A.E.Mueller and H.Patel, Nucl. Phys. B425 (1994) 471.

[5] B.Z.Kopeliovich and B.G.Zakharov, Phys. Rev. D44 (1991) 3466.

[6] N.N.Nikolaev, Comments on Nucl. Part. Phys. 21 (1992) 41.

[7] B.Z.Kopeliovich, J.Nemchik, N.N.Nikolaev and B.G.Zakharov, Phys. Lett. B309 (1993) 179 .

[8] B.Z.Kopeliovich, J.Nemchik, N.N.Nikolaev and B.G.Zakharov, Phys.Lett. B324 (1994) 469.

[9] J.Nemchik, N.N.Nikolaev and B.G.Zakharov, Phys.Lett. B341 (1994) 228.

[10] M.G.Ryskin, Z. Phys. C57 (1993) 89.

[11] S.J.Brodsky et al., Phys. Rev. D50 (1994) 3134.

[12] N.N.Nikolaev and B.G.Zakharov, Phys. Lett. B327 (1994) 157.

[13] V.N.Gribov, A.A.Migdal, Sov. J. Nucl. Phys. 8 (1969) 703

[14] A.Donnachie and P.V.L.Landshoff, Phys. Lett. B296 (1992) 227; Phys. Lett. B348 (1995) 213

[15] N.N.Nikolaev and B.G.Zakharov, Phys. Lett. B327 (1994) 149.

[16] J. Busenitz et al., Phys. Rev. D40 (1989) 40 and refrences therein.

[17] ZEUS Collab., M.Derrick et al., Elastic Photoproduction of $\omega, \phi$ and $\rho^{\prime}$ mesons at HERA, presented on Int. Europhys. Conf. on HEP, Brussels, July 27 - August 2, 1995, paper EPS-0389 
[18] NMC Collab., M.Arneodo, et al., Nucl.Phys. B 429 (1994) 503

[19] H1 Collab., T.Ahmed et al., Exclusive $\rho^{0}$ Production in Deep-inelastic Scattering Events at HERA, presented on Int. Europhys. Conf. on HEP, Brussels, July 27 - August 2, 1995, paper EPS-0490

[20] ZEUS Collab., M.Derrick et al., Z. Phys. C63 (1994) 391.

[21] ZEUS Collab., M.Derrick et al., DESY 95-143, submitted to Z. Phys. C (1995)

[22] ZEUS Collab., M.Derrick et al., Phys. lett. B356 (1995) 601

[23] EMC Collab., J.J.Aubert et al., Phys. Lett. B89 (1980) 267.

[24] L.Frabetti et al., Phys. Lett. B316 (1993) 197.

[25] EMC Collab., J.J.Aubert et al., Nucl. Phys. B213 (1983) 1;

J.Ashman et al.,Z. Phys. C39 (1988) 169.

[26] H1 Collab., T.Ahmed et al., Phys. Lett. B338 (1994) 507

[27] ZEUS Collab., M.Derrick et al., Phys. Lett. B350 (1995) 120

[28] EMC Collab., J.J.Aubert et al., Phys. Lett. B161 (1985) 203. 


\begin{tabular}{||l||c|c|c|c|}
\hline \hline parameter & $\rho^{0}$ & $\phi^{0}$ & $J / \Psi$ & $\Upsilon$ \\
\hline$R^{2}\left[\mathrm{fm}^{2}\right]$ & 1.370 & 0.690 & 0.135 & 0.015 \\
\hline$C$ & 0.360 & 0.530 & 1.130 & 1.990 \\
\hline$m_{q}[\mathrm{GeV}]$ & 0.150 & 0.300 & 1.300 & 5.000 \\
\hline \hline
\end{tabular}

Table 1: Values of the parameters $R^{2}, C$ and $m_{q}$ of the wave function of vector mesons [9]. 


\section{Figure captions:}

Fig. 1 The $Q^{2}$ dependence of the coefficient functions $g_{T, L}$ at $W=15 \mathrm{GeV}$ (dashed curve) and $W=150 \mathrm{GeV}$ (solid curve).

Fig. 2 The dipole size dependence of the dipole cross section extracted from the experimental data on photoproduction and electroproduction of vector mesons: the NMC data on $\phi^{0}$ and $\rho^{0}$ production [18], the EMC data on $J / \Psi$ production [23, 25], the E687 data on $J / \Psi$ production [24], the FNAL data on $\rho^{0}$ production [16], the ZEUS data on $\rho^{0}$ production [20, 21, 22], the ZEUS data on $\phi^{0}$ production [17], the H1 data on $\rho^{0}$

production [19] and the average of the H1 and ZEUS data on $J / \Psi$ production [26, 27]. The dashed and solid curve show the dipole cross section of the model [15, 9] evaluated for the c.m.s. energy $W=15$ and $W=70 \mathrm{GeV}$ respectively. The data points at HERA energies and the corresponding solid curve are multiplied by the factor 1.5. 


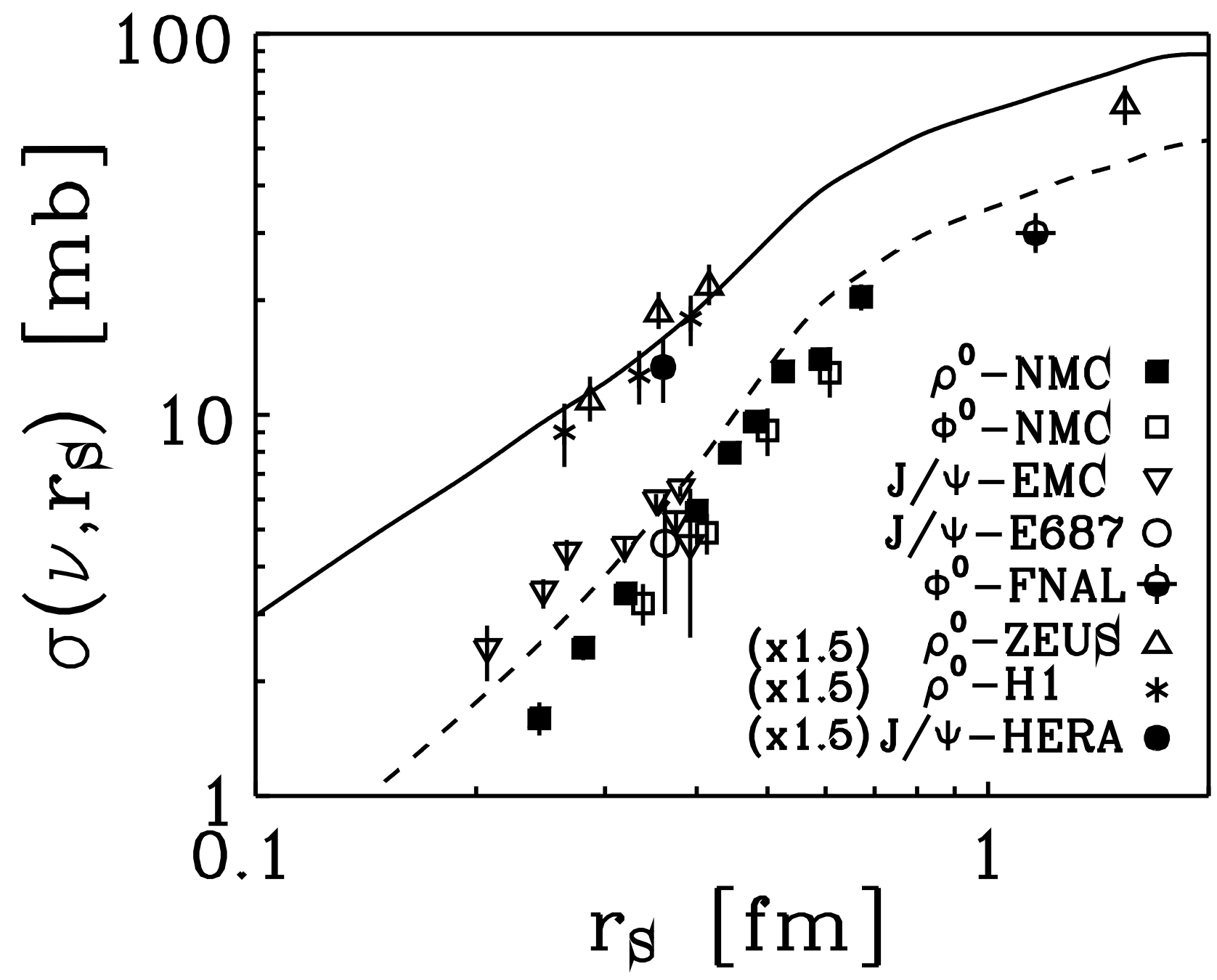




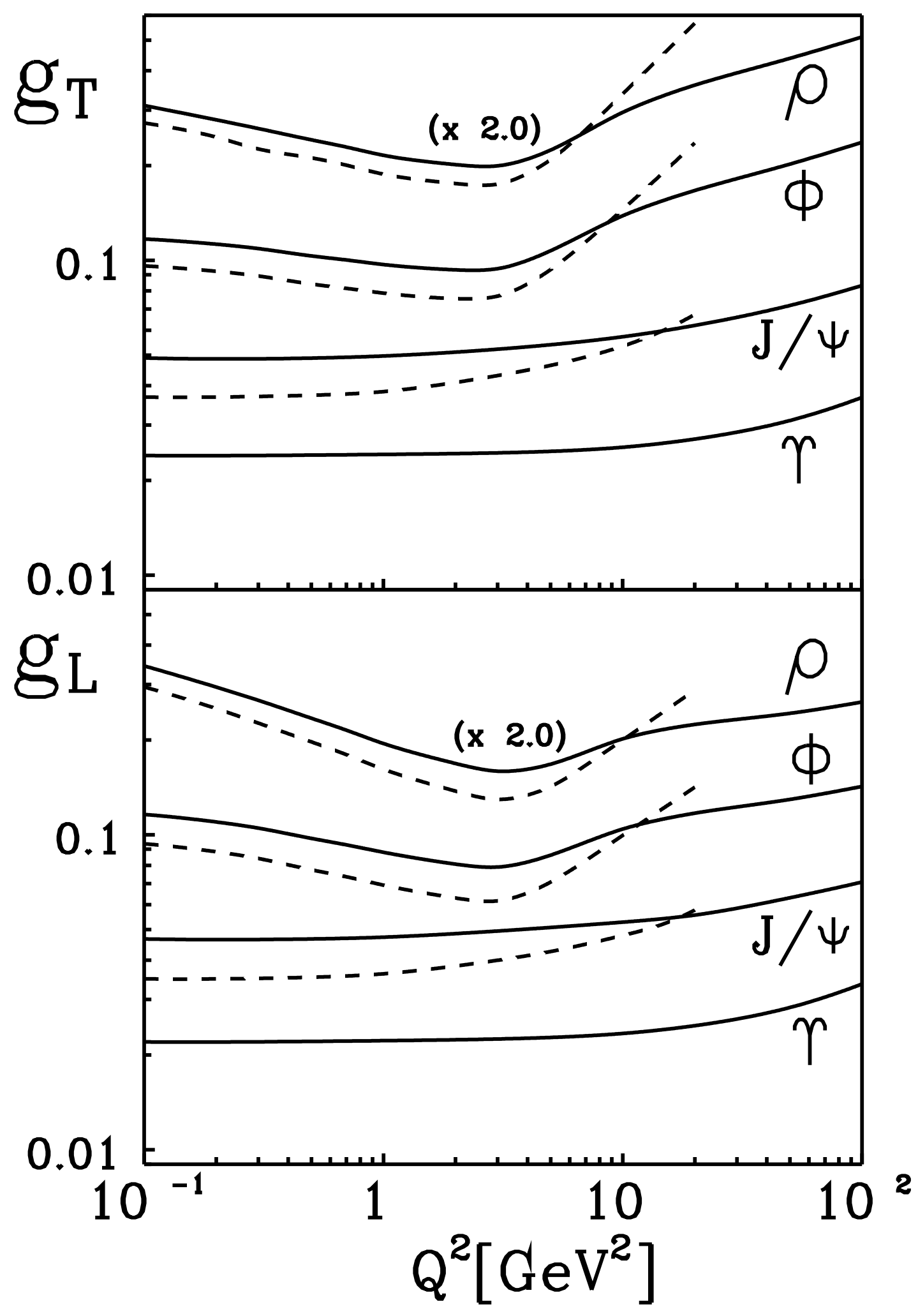

